\title{
An analytic exact form of Heaviside function
}

J. Venetis

Section of Mechanics,

Faculty of Applied Mathematics and Physical Sciences,

National Technical University of Athens

*Corresponding Author: johnvenetis4@gmail.com

\begin{abstract}
In this paper, the author obtains an analytic exact form of Heaviside function, which is also known as Unit Step function and constitutes a fundamental concept of the Operational Calculus.

In particulat, this function is explicitly expressed in a very simple manner by the aid of purely algebraic representations. The novelty of this work is that the proposed explicit formula is not performed in terms of non - elementary special functions, e.g. Dirac delta function or Error function and also is neither the limit of a function, nor the limit of a sequence of functions with point wise or uniform convergence. Hence, it may be much more appropriate and useful in the computational procedures which are inserted into Operational Calculus techniques and other engineering practices.
\end{abstract}

\section{Keywords}

Heaviside function, single valued function, real variable, integer part

\section{Introduction}

The Heaviside function, or unit step function, which is usually notated by the symbols $\mathrm{H}$ or $\mathrm{u}$, is a discontinuous single - valued function, the value of which is zero for negative argument and equal to unity for positive argument [1]. This function was introduced by Oliver Heaviside, who was an important pioneer in the study of electronics and also made a remarkable contribution to the field of Operational Calculus [2]. A very important characteristic of this function is that is able to be represented either as a piecewise constant function or as a generalized function [1,3]. The unit step function is mainly used in the calculation processes of Control Theory and signal processing in order to represent a signal which switches on at a specified time and stays switched on indefinitely. It is also applied along with its derivative, i.e. Dirac delta function, in structural engineering to describe various types of structural loads, e.g. off - axis four point bending of simply supported or fully constrained beams. Hence, it is very useful for the necessary calculations dealing with conceptual and embodiment design procedures from the engineering viewpoint.

Meanwhile, there are many smooth analytic approximations to the unit step function as it can be seen in the literature [4,5,6]. Besides, Sullivan et al [7] obtained a linear algebraic approximation to this function by means of a linear combination of exponential functions. However, the majority of all these approaches lead to closed - form representations consisting of non - elementary special functions, e.g. Logistic function, Hyperfunction, or Error function and also most of its algebraic exact forms are expressed in terms generalized integrals or infinitesimal terms, something that complicates the related computational procedures. In Ref. [8] proposed an analytic exact form of the Unit Step Function as a sum of two inverse tangent functions however, according to this approach, the singularity structure was left ambiguous. Unfortunately, the same problem will remain if one adopts a similar analytical approach appearing in Ref. [9] to approximate Heaviside function. Also, one may point out that a serious shortcoming of such formulae is that the inverse trigonometric functions do not have unique definitions.

Finally, another elegant approximation to Heaviside function in the form of a summation of two logarithmic functions was carried out by Murphy in Ref. [10].

In the present work, we introduce a single - valued function which is proved to be synonymous to the Unit Step Function. This formula constitutes a purely algebraic representation and does not either contain 
generalized integrals or any infinitesimal quantities. In this framework it may have good prospects towards the computational procedures that concern the applications of Heaviside function in Operational Calculus, as well as in engineering practices.

\section{Towards an analytical expression of Heaviside function}

Let us introduce the following single valued real function

$f: R \rightarrow R$ such that

$f(x)=1-\left[\frac{1}{\mathrm{n}-\left[\mathrm{e}^{x}+\mathrm{n}\right] \cdot\left[\frac{\mathrm{n}}{\left[\mathrm{e}^{x}+\mathrm{n}\right]}\right]+1}\right] \forall n \in N^{*}$

\section{Claim}

The function $f$ is identical with the Unit Step Function over its domain of definition i.e. over the set $R$.

\section{Proof}

i) Let $x$ be a strictly negative real variable. To show that $f(x)$ vanishes, it is enough to prove that the integer part of the fraction $\frac{1}{\mathrm{n}-\left[\mathrm{e}^{x}+\mathrm{n}\right] \cdot\left[\frac{\mathrm{n}}{\left[\mathrm{e}^{x}+\mathrm{n}\right]}\right]+1}$, equals unity $\forall n \in N^{*}$.

When $x<0$ it implies that $0<\mathrm{e}^{x}<1$ and therefore

$n<\mathrm{e}^{x}+\mathrm{n}<\mathrm{n}+1$

Thus it follows

$\left[\mathrm{e}^{x}+\mathrm{n}\right]=\mathrm{n}$

or equivalently

$\frac{n}{\left[\mathrm{e}^{x}+\mathrm{n}\right]}=1$

Evidently, $\frac{n}{\left[\mathrm{e}^{x}+\mathrm{n}\right]} \in N^{*}$.

Thus one infers

$\left[\frac{\mathrm{n}}{\left[\mathrm{e}^{x}+\mathrm{n}\right]}\right]=\frac{n}{\left[\mathrm{e}^{x}+\mathrm{n}\right]}$

In this context, Eqn. (1) yields 
$f(x)=1-\left[\frac{1}{1}\right]=1-1=0$

ii) Let $x$ be equal to zero. Evidently $\mathrm{e}^{x}=\mathrm{e}^{0}=1$

Here one may easily observe that the quotient $\frac{\mathrm{n}}{[1+\mathrm{n}]}$ is not a rational variable.

Then $\forall \mathrm{n} \in N \exists \mathrm{a} \in Q \cap(0,1)$ such that

$\frac{\mathrm{n}}{[1+\mathrm{n}]}=\left[\frac{\mathrm{n}}{[1+\mathrm{n}]}\right]+\mathrm{a}$

or equivalently

$n=[1+\mathrm{n}] \cdot\left[\frac{\mathrm{n}}{[1+\mathrm{n}]}\right]+[1+\mathrm{n}] \cdot \mathrm{a} \Leftrightarrow$
$n-[1+\mathrm{n}] \cdot\left[\frac{\mathrm{n}}{[1+\mathrm{n}]}\right]+1=[1+\mathrm{n}] \cdot \mathrm{a}+1$

Moreover, the following inequality holds

$[1+\mathrm{n}] \cdot \mathrm{a}+1>1$

and therefore

$0<\frac{1}{[1+\mathrm{n}] \cdot \mathrm{a}+1}<1$

Thus one infers

$\left[\frac{1}{[1+n] \cdot a+1}\right]=0$

Eqn. (10) can be combined with eqn. (7) and eqn. (1) to yield

$f(0)=1-0=1$

iii) Let $x$ be a strictly positive real variable

Then $\forall \mathrm{n} \in N, \forall \mathrm{x} \in(0,+\infty) \exists \mathrm{b} \in(0,1)$ such that

$\frac{\mathrm{n}}{\left[\mathrm{e}^{x}+\mathrm{n}\right]}=\left[\frac{\mathrm{n}}{\left[\mathrm{e}^{x}+\mathrm{n}\right]}\right]+\mathrm{b}$

and therefore

$n=\left[\frac{\mathrm{n}}{\left[\mathrm{e}^{x}+\mathrm{n}\right]}\right] \cdot\left[\mathrm{e}^{x}+\mathrm{n}\right]+\mathrm{b} \cdot\left[\mathrm{e}^{x}+\mathrm{n}\right] \Leftrightarrow$ 
$n-\left[\frac{\mathrm{n}}{\left[\mathrm{e}^{x}+\mathrm{n}\right]}\right] \cdot\left[\mathrm{e}^{x}+\mathrm{n}\right]+1=\mathrm{b} \cdot\left[\mathrm{e}^{x}+\mathrm{n}\right]+1$

Moreover

$\mathrm{b} \cdot\left[\mathrm{e}^{x}+\mathrm{n}\right]+1>1$

and therefore

$0<\frac{1}{\mathrm{~b} \cdot\left[\mathrm{e}^{x}+\mathrm{n}\right]+1}<1$

Thus one may deduce that

$\left[\frac{1}{\mathrm{~b} \cdot\left[\mathrm{e}^{x}+\mathrm{n}\right]+1}\right]=0$

Eqn. (16) can be combined with eqn. (13) and eqn. (1) to yield

$f(x)=1-0=1$

\section{Discussion}

In the previous unit, we gave a rigorous proof, that all the elements of the set of single valued functions which are defined by eqn. (1) for any nonzero natural number $n$ coincide with the Heaviside function over the set of real numbers. Here, one may pinpoint that to define $\mathrm{H}(0)$ as $1 / 2$ something that is taken for granted in many explicit approximations of this function, cannot be in consensus with the proposed formula in the present work. On the contrary, according to the performed algebraic representation it was definitely shown that $f(0)=1$

\section{Conclusions}

The objective of this work was to propose an analytic exact form of the unit step function. Specifically, the author utilized the properties of the integer part of a real variable to construct a singe valued function which coincides with Heaviside function over the set of real numbers. Nevertheless, since this formula constitutes a purely algebraic representation and does not contain generalized integrals or any infinitesimal quantities, may have good prospects towards the computational procedures that concern the applications of the unit step function in Operational Calculus, as well as in many engineering practices.

\section{References}

[1] Abramowitz, M. and Stegun, I. A. (1972). Handbook of Mathematical Functions with Formulas, Graphs and Mathematical Tables, Dover Publications INC

[2] Hunt, B. J. (2012). Oliver Heaviside. Phys. Today, 65(11), 48.

[3] Bracewell, R. (2000). Heaviside's Unit Step Function,H(x). The

Fourier Transform and its Applications, McGraw - Hill

[4] Berg L. (1967). Introduction to the Operational Calculus, North - Holland Publishing Company 
[5] Kanwal, R. P. (1998). Generalized Functions Theory and Technique: Theory and Technique. 2nd ed. Boston, MA: Birkhäuser

[6] Spanier, J. and Oldham, K. B. (1987). The Unit-Step u(x-a) and Related Functions Ch. 8 from: An Atlas of Functions. Washington, DC: Hemisphere, 63-69.

[7] Sullivan J. Crone L. Jalickee J. (1980) Approximation of the Unit Step Function by a Linear Combination of Exponential

Functions, Journal of. Approximation Theory, 28, 299 - 308

[8] J. Venetis, An analytic exact form of the unit step function, Mathematics and Statistics 2(7) (2014), 235-237.

[9] J. Venetis and E. Sideridis, Study of asymmetric elastic beams in off-axis four-point bending, International Review of Aerospace Engineering 8(6) (2015), 185-197.

[10] M. Kyle Murphy, EXPLICIT FORMS OF DISCONTINUOUS FUNCTIONS, THE DIRAC DELTA, AND IRREDUCIBLE FORMS (preprint)

https://www.academia.edu/11704122/Explicit Forms of Discontinuous Functions the Dirac Delta and Irreduci ble_Forms 